\title{
Investigating rare pathogenic/likely pathogenic exonic variation in bipolar disorder
}

\author{
Xiaoming Jia ${ }^{1}$. Fernando S. Goes $\mathbb{1}^{2} \cdot$ Adam E. Locke $\mathbb{1}^{3} \cdot$ Duncan Palmer $\mathbb{1}^{4} \cdot$ Weiqing Wang $\mathbb{1}^{5}$. \\ Sarah Cohen-Woods ${ }^{6,7} \cdot$ Giulio Genovese $\mathbb{1}^{4} \cdot$ Anne U. Jackson $\mathbb{1}^{8} \cdot$ Chen Jiang $^{9} \cdot$ Mark Kvale $^{10} \cdot$ Niamh Mullins $^{11}$. \\ Hoang Nguyen $\mathbb{1}^{5} \cdot$ Mehdi Pirooznia $\mathbb{1}^{12} \cdot$ Margarita Rivera ${ }^{7,13} \cdot$ Douglas M. Ruderfer $^{14} \cdot$ Ling Shen $^{9} \cdot$ Khanh Thai $^{9} \cdot$ \\ Matthew Zawistowski $\mathbb{D}^{8} \cdot$ Yongwen Zhuang ${ }^{8} \cdot$ Gonçalo Abecasis $\mathbb{1}^{8} \cdot$ Huda Akil ${ }^{15} \cdot$ Sarah Bergen $\mathbb{D}^{16}$. \\ Margit Burmeister $\mathbb{D}^{15,17,18,19}$. Sinéad Chapman ${ }^{4} \cdot$ Melissa DelaBastide ${ }^{20} \cdot$ Anders Juréus $^{16} \cdot$ Hyun Min Kang $^{8}$.

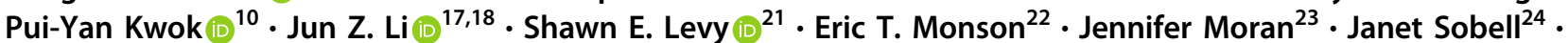

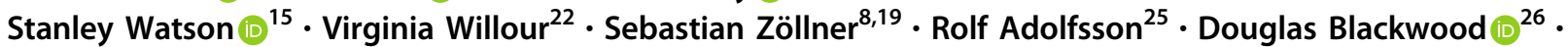 \\ Michael Boehnke $\mathbb{1}^{8} \cdot$ Gerome Breen $^{7,27} \cdot$ Aiden Corvin $^{28} \cdot{\text { Nick } \text { Craddock }^{29} \text { • Arianna DiFlorio }}^{29}$. \\ Christina M. Hultman ${ }^{16}$. Mikael Landen $\mathbb{1}^{16,30} \cdot$ Cathryn Lewis $\mathbb{D}^{7,31} \cdot$ Steven A. McCarroll $\mathbb{D}^{32}$. \\ W. Richard McCombie ${ }^{20} \cdot$ Peter McGuffin ${ }^{7}$. Andrew Mclntosh $\mathbb{D}^{26,33}$. Andrew McQuillin $\mathbb{D}^{34} \cdot$ Derek Morris $\mathbb{D}^{28,35}$. \\ Richard M. Myers ${ }^{21} \cdot$ Michael O'Donovan (10) ${ }^{29} \cdot$ Roel Ophoff ${ }^{36,37} \cdot$ Marco Boks $\mathbb{1}^{37} \cdot$ Rene Kahn $\mathbb{( 1 0}^{38}$. \\ Willem Ouwehand $\mathbb{1}^{39}$. Michael Owen $\mathbb{1}^{29}$. Carlos Pato ${ }^{24,40}$. Michele Pato ${ }^{24,41}$. Danielle Posthuma ${ }^{42,43}$. \\ James B. Potash $\mathbb{1}^{2}$ - Andreas Reif $\mathbb{1}^{44} \cdot$ Pamela Sklar $^{5}$ - Jordan Smoller ${ }^{4,45}$ - Patrick F. Sullivan ${ }^{46}$. \\ John Vincent $\mathbb{D}^{47,48}$. James Walters ${ }^{29} \cdot$ Benjamin Neale $\mathbb{D}^{4,49} \cdot$ Shaun Purcell $\mathbb{D}^{50,51} \cdot$ Neil Risch $^{10}$. \\ Catherine Schaefer $\mathbb{1}^{9} \cdot$ Eli A. Stahl $\mathbb{1}^{5} \cdot$ Peter P. Zandi $\mathbb{1}^{2} \cdot$ Laura J. Scott $\mathbb{1}^{8}$
}

Received: 2 November 2019 / Revised: 14 December 2020 / Accepted: 16 December 2020 / Published online: 22 January 2021 (c) The Author(s) 2021. This article is published with open access

\begin{abstract}
Bipolar disorder (BD) is a serious mental illness with substantial common variant heritability. However, the role of rare coding variation in BD is not well established. We examined the protein-coding (exonic) sequences of 3,987 unrelated individuals with $\mathrm{BD}$ and 5,322 controls of predominantly European ancestry across four cohorts from the Bipolar Sequencing Consortium (BSC). We assessed the burden of rare, protein-altering, single nucleotide variants classified as pathogenic or likely pathogenic (P-LP) both exome-wide and within several groups of genes with phenotypic or biologic plausibility in BD. While we observed an increased burden of rare coding P-LP variants within 165 genes identified as BD GWAS regions in 3,987 $\mathrm{BD}$ cases (meta-analysis $\mathrm{OR}=1.9,95 \% \mathrm{CI}=1.3-2.8$, one-sided $p=6.0 \times 10^{-4}$ ), this enrichment did not replicate in an additional 9,929 BD cases and 14,018 controls ( $\mathrm{OR}=0.9$, one-side $p=0.70)$. Although $\mathrm{BD}$ shares common variant heritability with schizophrenia, in the BSC sample we did not observe a significant enrichment of P-LP variants in SCZ GWAS genes, in two classes of neuronal synaptic genes (RBFOX2 and FMRP) associated with SCZ or in loss-of-function intolerant genes. In this study, the largest analysis of exonic variation in BD, individuals with BD do not carry a replicable enrichment of rare P-LP variants across the exome or in any of several groups of genes with biologic plausibility. Moreover, despite a strong shared susceptibility between BD and SCZ through common genetic variation, we do not observe an association between BD risk and rare P-LP coding variants in genes known to modulate risk for SCZ.
\end{abstract}

These authors contributed equally: Xiaoming Jia, Fernando Goes, Adam E. Locke, Duncan Palmer, Weiqing Wang

These authors jointly supervised this work: Shaun Purcell, Neil Risch, Catherine Schaefer, Eli A Stahl, Peter P Zandi, Laura J. Scott

Supplementary information The online version of this article (https:// doi.org/10.1038/s41380-020-01006-9) contains supplementary material, which is available to authorized users.

Peter P. Zandi pzandi1@jhmi.edu

$\bowtie$ Laura J. Scott ljst@umich.edu

Extended author information available on the last page of the article 
Table 1 Bipolar disorder sequencing cohorts.

\begin{tabular}{|c|c|c|c|c|c|c|c|c|}
\hline Study & Ethnicity & Total $N$ & $\begin{array}{l}\text { Case/ } \\
\text { Control } N\end{array}$ & Female $(\%)$ & $\begin{array}{l}\text { B1D/B2D/SAB/ } \\
\text { NOS/UNK }(\%)\end{array}$ & P-LP SNVs & Exonic SNVs & $\begin{array}{l}\text { Exonic coverage: } \\
\text { median }(25-75 \%)\end{array}$ \\
\hline BRIDGES $^{\mathrm{a}}$ & US-Caucasian & 3,556 & $1712 / 1844$ & 32.4 & $100 / 0 / 0$ & 4,812 & 680,910 & $8(6-11)$ \\
\hline RareBLISS & US-Caucasian & 2,000 & $961 / 1039$ & 53.0 & $90.8 / 0 / 9.2$ & 3,394 & 465,504 & $78(42-101)$ \\
\hline Sweden & $\begin{array}{l}\text { Swedish- } \\
\text { Caucasian }\end{array}$ & 2,787 & $831 / 1956$ & 53.8 & $\begin{array}{l}65.8 / 30.3 / 0.4 / 1.7 / \\
1.8\end{array}$ & 1,945 & 328,066 & $28(24-37)$ \\
\hline KPNC-EUR & US-Caucasian & 384 & $192 / 192$ & 71.6 & $100 / 0 / 0$ & 511 & 108,307 & $34(22-51)$ \\
\hline KPNC-AFR & $\begin{array}{l}\text { US-African } \\
\text { American }\end{array}$ & 191 & $96 / 95$ & 71.2 & $100 / 0 / 0$ & 308 & 132,872 & $39(25-57)$ \\
\hline KPNC-LAT & US-Latino & 198 & $98 / 100$ & 72.7 & $100 / 0 / 0$ & 298 & 101,368 & $40(26-60)$ \\
\hline KPNC-EAS & US-East Asian & 193 & $97 / 96$ & 63.7 & $100 / 0 / 0$ & 334 & 88,884 & $39(26-58)$ \\
\hline All & & 9,309 & $3987 / 5322$ & 47.1 & $\begin{array}{l}90.6 / 6.3 / 2.3 \\
0.4 / 0.4\end{array}$ & 9,883 & $1,328,324$ & \\
\hline
\end{tabular}

We examined protein-coding (exome) sequence data in 9309 individuals of predominantly European ancestry from four independent studies. Cases and controls were recruited at $\sim 1: 1$ ratio except in the Sweden cohort ( 1:2 cases to controls). The Kaiser Permanente Northern California (KPNC) cohort was comprised of four race/ethnicity groups: 40\% non-Hispanic white, 20\% African American, 20\% Latino, and 20\% East Asian. The number of unique exon variants (including exonic, splicing, and 3' and 5' UTR regions) is listed for each cohort and across studies.

$S N V$ single nucleotide variant, WES whole-exome sequencing, BRIDGES Bipolar Research in Deep Genome and Epigenome Sequencing Study, RareBLISS Rare Bipolar Loci Identification through Synaptome Sequencing, EUR European ancestry, AFR African American ancestry, LAT Latino ancestry, EAS East Asian ancestry, B1D bipolar 1 disorder, $B 2 D$ bipolar 2 disorder, $S A B$ Schizoaffective disorder, bipolar type, NOS No otherwise specified, Miss missing specific diagnosis.

${ }^{a}$ Whole-genome sequencing was performed in BRIDGES. Exome sequencing was performed in all other BSC cohorts.

\section{Introduction}

Bipolar disorder (BD) is a serious mental illness affecting more than 48 million adults worldwide with a lifetime prevalence of $1-3 \%[1,2]$. BD is characterized by extreme episodes of mood elevation and depression, along with disturbances in thinking and behavior that often result in significant disability [3]. The high lifetime morbidity of BD and frequent suboptimal outcomes with available treatments present an urgent need to better understand the pathogenesis of BD. Prior studies have suggested a role for dysregulated neuronal signaling and synaptic plasticity [4-7], as well as pathways that regulate neurotransmitter function, neuronal development, and oxidative stress [8, 9]. However, a comprehensive characterization of biologic mechanisms in BD remains elusive. A better understanding is critical for development of effective therapies and personalized care.

BD is highly heritable (59-93\%) within families, and monozygotic twins show higher concordance rates (40-80\%) than dizygotic twins (5-30\%) [10-14]. Genomewide association studies (GWAS) have identified over 30 loci that contribute to BD susceptibility, implicating genes that encode ion channels, neurotransmitter transporters, and synaptic components [15-18]. Genes implicated by GWAS might also harbor rare variants [19-21], however this has not been studied in BD. Whole-exome sequencing (WES) in BD families has examined the role of rare exonic variation influencing BD risk. [22-26]. However, these studies have been small (discovery cohort $<200$ BD cases) [22-25], are single-cohort studies [22, 25], and only provide modest evidence implicating rare coding variants $[23,24,26]$. Overall, it remains unclear whether rare genetic variation significantly influences risk of $\mathrm{BD}$.

$\mathrm{BD}$ shares clinical features and genetic susceptibility with schizophrenia (SCZ) [12, 18, 27-29], particularly through common genetic variation. In addition to the observation that $\mathrm{BD}$ and $\mathrm{SCZ}$ share a high genetic correlation due to common variation [30], the SCZ polygenic risk score (PRS) is associated with psychosis in $\mathrm{BD}$, and the BD PRS is associated with manic behavior in schizophrenia $[28,31]$. Studies of rare variation show that individuals with SCZ carry a higher burden of ultra-rare protein-altering variants across the exome, particularly in genes that are evolutionarily conserved, in genes that affect neuronal synaptic function, and that have been implicated through studies of de novo variation in SCZ and autism spectrum disorder [32-36]. Rare variants in these gene classes have not been systematically examined in BD. Despite the shared susceptibility between BD and SCZ through common genetic variation, it remains unknown whether rare variants in genes implicated in SCZ contribute to BD.

This study aims to test whether rare, functional proteinaltering variation impacts susceptibility to BD. To this end, we examined the protein-coding sequences (exomes) of 3,987 individuals with BD and 5,322 healthy controls of predominantly European ancestry recruited across four studies to address the following questions: do individuals 
with BD carry a greater burden of rare functional coding variants (1) in their exomes overall; (2) in genes implicated by common variant GWAS in BD; and (3) in genes implicated through common and rare variant studies in SCZ, including neuronal synaptic and loss-of-function intolerant genes. We subsequently attempted replication of positive findings in an additional 9,929 individuals with BD and 14,018 matched controls.

\section{Methods}

\section{Cohorts}

The study protocol for the combined analysis was approved by the Johns Hopkins University Institutional Review Board. All human studies were approved by each respective institutional ethics review committee, and all participants provided written informed consent. We examined the exomes of 3,987 individuals with BD (3,615 with bipolar 1 disorder (B1D), 252 with bipolar 2 disorder (B2D), 91 with schizoaffective disorder bipolar type (SAB)) and 5,322 unaffected individuals from four case-control cohorts of predominantly European ancestry that comprise the Bipolar Sequencing Consortium (BSC) (Table 1, Figs. S1, S2, and Supplemental Methods). These included 1,712 cases and 1,844 controls from the Bipolar Research in Deep Genome and Epigenome Sequencing (BRIDGES) study [37], 961 cases and 1,039 controls from the Rare Bipolar Loci Identification Through Synaptome Sequencing (RareBLISS) study (NIMH Genetics Initiative repository) [26], 831 cases and 1,956 controls from Sweden (Swedish National Hospital Discharge Register and population-based registries), and 483 cases and 483 matched controls from the Kaiser Permanente Northern California (KPNC) cohort (Multiethnic Genome-wide Study of Bipolar1 Disorder and Resource for Genetic Epidemiology Research in Adult Health and Aging (GERA) cohort [38]). All studies were comprised of individuals of European ancestry except for KPNC, which included individuals of European (EUR, 192 cases, 192 controls), African American (AFR, 96 cases, 95 controls), Latino (LAT, 98 cases, 100 controls), and East Asian (EAS, 97 cases, 96 controls) ancestry.

\section{Sequencing and quality control}

Whole-genome sequencing (WGS) was performed in the BRIDGES cohort using the Illumina HiSeq 2500 system. Library preparation was performed using Nimblegen SeqCap EZ Exome (RareBLISS and KPNC) or Agilent SureSelect Human All Exon v2 kit (Sweden), and wholeexome sequencing (WES) was performed using Illumina
HiSeq 2000 or 2500 systems in RareBLISS, Sweden, and KPNC. Paired sequence reads were aligned to the human reference build hg19 using BWA [39]. Variant calling was performed using the GotCloud sequence analysis pipeline [40] for WGS data in BRIDGES, and joint variant calling was performed using the Genome Analysis ToolKit (GATK) [41] for WES data within each of RareBLISS, Sweden, and KPNC. Genotypes with low sequence coverage or poor call quality were removed. Samples that were identified as population outliers, duplicates or relatives, or that failed study-specific sequencing metrics were removed. Variants with high missingness across samples, poor average genotyping quality, or found to significantly deviate from Hardy-Weinberg equilibrium were removed. A full description of sequencing and data quality control is provided in Supplemental Materials.

\section{Variant selection and annotation}

Analysis was limited to single nucleotide variants (SNVs) that are bi-allelic and whose minor allele matched the alternative (non-reference) allele. Annotation of all variants identified from exome and genome sequencing was done using ANNOVAR, and pathogenicity was assigned according to 2015 American College of Medical Genetics (ACMG) criteria using InterVar, a computational implementation of expert panel recommendations for clinical interpretation of genetic variants (ACMG 2015 criteria) [42-44]. ACMG classification categories include pathogenic (P), likely pathogenic (LP), variant of uncertain significance, likely benign, and benign. Variants that were rare (maximum population-specific minor allele frequency $[\mathrm{MAF}]<1 \%$ in the Genome Aggregation Database [gno$\mathrm{mAD}][45,46])$, protein-altering (missense, splice site, stopgain, startloss, stoploss (none observed), and classified as pathogenic or likely pathogenic by InterVar were retained for analysis.

\section{Gene-level burden testing}

To assess whether individuals with BD carry a greater burden of rare pathogenic or likely pathogenic (P-LP) variants in individual genes, we performed Firth logistic regression on the number of P-LP alleles within the gene per individual (adjusted for sex and at least five ancestry principal components) for each of seven groups (BRIDGES, RAREBLISS, Sweden, KPNC-EUR, KPNC-AFR, KPNC-LAT, KPNCEAS), followed by a meta-analysis across groups using the weighted Z-score method. We calculated a one-sided $p$ value for enrichment of P-PL variants in BD cases from the meta-analysis Z-score, and used a Holm-adjusted $p$ value $<$ 0.05 to report significant associations. 


\section{Gene set selection}

We assessed whether exomes of BD patients are enriched for rare functional protein-altering variants in genes implicated by the largest BD GWAS meta-analysis [18]. We defined the following three BD-related gene sets: 165 genes identified by physical proximity to variants in linkage disequilibrium $\left(r^{2}>0.1\right)$ with BD GWAS-associated variants (BD GWAS), 153 genes identified using the software package MAGMA (Multi-marker Analysis of GenoMic Annotation) [47] on BD GWAS data (BD MAGMA), and 81 genes identified through the intersection of the above two gene sets (BD MAGMA-GWAS). Given that BD shares genetic susceptibility with schizophrenia (SCZ) [12, 18, 27-29], particularly through common genetic variation, we examined genes implicated in a large schizophrenia GWAS (CLOZUK+PGC2) [48] by defining the following three gene sets: 623 genes identified by variants in linkage disequilibrium $\left(r^{2}>0.1\right)$ with schizophrenia GWAS-associated variants (SCZ GWAS), 550 genes identified using MAGMA on schizophrenia GWAS data (SCZ MAGMA), and 342 genes identified through the intersection of these two gene sets (SCZ MAGMA-GWAS). Given the strong impact of rare variant burden on SCZ susceptibility and the partial clinical overlap between BD and SCZ [34, 48-50], we also examined three gene sets previously implicated in SCZ rare variant risk: 3,055 synaptic genes whose mRNA are bound by the splicing factor RBFOX2 [32, 51] (Synaptic-RBFOX2), 1,033 synaptic genes whose mRNA are bound by FMRP [32, 52] (Synaptic-FMRP), and 3,230 genes classified as loss-of-function (LOF) intolerant from the large ExAC dataset [45] (LOF-intolerant).

\section{Gene set burden testing}

We used two statistical methods to evaluate whether BD cases carry a higher burden of rare functional protein-altering variants (described above) than controls for each candidate gene set. First, we performed meta-analysis of Firth logistic regressions from each of seven groups described above. Second, we performed Cochran-Mantel-Haenszel (CMH) chi-square tests on the P-LP count in cases and controls across studies, and determined the empirical (one-sided) $p$ value for enrichment of P-PL variants in BD cases by comparing this $\mathrm{CMH}$ chi-square statistic to a null distribution generated from a random selection of an equivalent number of genes in 10,000 simulations. We performed these random simulations to control for potential confounding due to variability in sample selection and sequencing methods between cases and controls within each study. We conducted all statistical analyses in R [53]. See Supplemental Methods for full burden testing methods.

\section{Replication}

We examined exome sequence data in an independent cohort of 9,929 individuals with BD and 14,018 matched controls from the Bipolar Exomes (BipEx) collection (see Supplemental Materials). Of 3,639 BD cases with known subtypes, 2,684 were classified as B1D, and 955 were classified as B2D. After WES variant calling and quality control, we annotated exonic variants using InterVar, and extracted all rare (gnomAD maximum population $\mathrm{MAF}<1 \%$ ), protein-altering (missense, splice site, stopgain, startloss) SNVs that were classified as P or LP, as was done for the BSC studies. Statistical analysis was the same as used in the BSC analysis. Specifically, we examined the burden of rare P-LP variants in BD cases versus controls, and in B1D cases versus controls, within the three BD gene sets (BD GWAS, BD MAGMA, BD MAGMA-GWAS). We performed Firth logistic regression on the number of rare P-LP alleles per individual (adjusted for sex, 10 principal components, and the total burden of non-reference alleles) within each of six cohorts that comprised the BipEx replication sample (US, UK, Sweden-Umea, Sweden-Karolinska, Netherlands, and Germany), followed by a metaanalysis across cohorts using the weighted Z-score method.

\section{PRS analysis of common (GWAS) variants}

Except for BRIDGES, which used genotypes from WGS, studies used previously published GWAS array data from European-descent individuals imputed into the $1000 \mathrm{Gen}-$ omes or 1000 Genomes + Haplotype Reference Consortium reference panels to select variants for inclusion in polygenic risk score analysis $[18,38,54-58]$. We identified the set of variants present and well imputed (imputation $R^{2}$ or info score $>0.5$ ) and with MAF $>0.01$ in all studies and limited to the European ancestry sample in KPNC. We further filtered variants where any individual cohort frequency deviated from the mean frequency by $>0.2$, resulting in a final set of 2,855,373 SNPs for building polygenic scores.

To generate SNP sets and per SNP weights for building polygenic scores independent from the data in the current study, we started with association results from studies that participated in recent PGC2 analyses for bipolar disorder [18] and schizophrenia [59], then removed all studies with participants overlapping with BSC study samples and re-ran case-control association meta-analysis. These modified meta-analyses included roughly 11,000 cases and 17,000 controls for bipolar disorder and 19,000 cases and 28,000 controls for schizophrenia. In both the bipolar and schizophrenia association results, we extracted the subset of SNPs overlapping the high quality BSC variants described above and identified sets of variants to include in PRS analysis by 


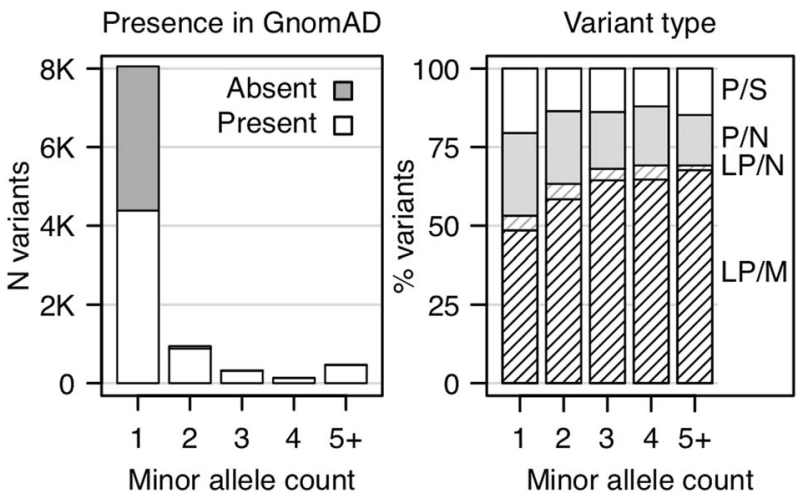

Fig. 1 P-LP variant distribution. Minor allele counts for variants classified as P or LP from 9,309 individuals in the BSC cohort show that $81 \%$ of P-LP variants are singleton mutations. $38 \%$ of P-LP variants are not present in gnomAD. $44 \%$ of such variants are classified as P (predominantly splice site and nonsense variants), and $56 \%$ are classified as LP (predominantly missense variants). P pathogenic, LP likely pathogenic, $\mathrm{S}$ Splice site, $\mathrm{N}$ nonsense, $\mathrm{M}$ missense, ACMG American College of Medical Genetics, GnomAD Genome Aggregation Database.

clumping all SNPs based on LD (1MB windows and $r^{2}>$ 0.1) using 1000 Genomes European individuals as the reference sample. For each trait, we selected variants at three different $p$ value thresholds $(P<0.01,<0.05$, and $<0.1)$ to determine the effect of threshold on the strength of the PRS association with bipolar disorder. Each study then generated a PRS score for each individual based on genotype dosages and using $\log (\mathrm{OR})$ from the restricted PGC2 analyses as weights. Each group then tested for association using logistic regression of the PRS on bipolar case-control status in their BSC GWAS/WGS data adjusting for sex, genotype-based principal components, and where necessary, genotype batch. We combined results from all studies using inverse variance-weighed meta-analysis.

Code used in the analysis of the BSC samples is available at: http://metamoodics.org/bsc/consortium/bsc-casecontrol-workgroup

\section{Results}

Exome analysis in 3,987 individuals with BD (91\% B1D, $6 \% \mathrm{~B} 2 \mathrm{D}$, and $2 \% \mathrm{SAB}$ ) and 5,322 unaffected individuals (Table 1, Table S1, Fig. S1) identified 1,328,324 exonic SNVs that passed quality control, of which 792,375 were rare (gnomAD maximum population $\mathrm{MAF}<1 \%$ ) and protein-altering (primarily missense, splice-site, and stopgain); 9,883 (0.7\%) of exonic SNVs were classified as pathogenic (P) or likely pathogenic (LP) using InterVar [42] (Table S2). Variants classified as pathogenic included 2,629 stop-gain and 1,963 splice-site altering SNVs affecting 2,076 genes. Variants classified as likely pathogenic included 5,152 missense, 463 stop-gain, and 1 stop-loss affecting
1,173 genes (Table S2). On average, each individual carried 0.72 pathogenic and 1.2 likely pathogenic SNVs across the exome. Most $(81 \%)$ of P-LP variants were singleton mutations, and a significant proportion (38\%) of such variants were not previously reported in the large gnomAD database (Fig. 1).

The exome-wide burden of rare synonymous variants did not differ between BD cases and controls (odds ratio [OR] $=1.00,95 \%$ confidence interval $[\mathrm{CI}]=0.9985-1.0004, p$ $=0.23$ ), suggesting an absence of exome-wide bias in detecting rare variants across a heterogeneous group of sequencing studies. We subsequently restricted our analysis to rare protein-altering variants classified as pathogenic or likely pathogenic (P-LP) (Table S2). Examination of genelevel burden of rare P-LP variants did not reveal any genes that passed exome-wide significance (Table S3). Likewise, the exome-wide P-LP burden also did not differ between $\mathrm{BD}$ cases and controls $(\mathrm{OR}=1.00,95 \% \mathrm{CI}=0.97-1.03, p$ $=0.39$ ), differing from sequencing studies of SCZ and developmental disorders of similar sample size showing an increased burden of rare coding variants across the exome $[32,42,49]$.

However, BD cases carried a higher burden of P-LP variants compared to controls within 165 bipolar GWAS genes (meta-analysis of Firth logistic regressions $\mathrm{OR}=$ $1.89,95 \% \mathrm{CI}=1.29-2.77$, one-sided $\left.p=6.0 \times 10^{-4}\right)$ and within 153 bipolar genes identified using MAGMA (metaanalysis $\mathrm{OR}=1.56,95 \% \mathrm{CI}=1.11-2.19$, one-sided $p=$ 0.0052) (Figs. 2, 3, Tables 2, S4 and S5). The relative burden of rare P-LP variants was higher within 81 genes representing the intersection of these two gene sets (metaanalysis $\mathrm{OR}=2.66,95 \% \mathrm{CI}=1.35-5.21$, one-sided $p=$ 0.0023) (Figs. 2, 3, Tables 2, S4 and S5). The CMH analysis results were consistent with those from meta-analysis of Firth logistic regressions described above and there was no evidence of heterogeneity in OR among the studies (Tables S4 and S5 for individual BSC study results). Metaanalysis results were also broadly consistent when subset to subjects of European ancestry (Tables S4 and S5).

Attempted replication in WES data from 9,929 BD cases and 14,018 controls (Table S6) from the BipEx collection did not reproduce the observed enrichment of rare P-LP variants within the BD GWAS (meta-analysis $\mathrm{OR}=0.94$, one-sided $p=0.70$ ), BD MAGMA ( $\mathrm{OR}=0.87, p=0.87$ ), or BD MAGMA-GWAS (OR $=0.64$, one-sided $p=0.95$ ) gene sets (Fig. 3, Table S7). Similarly, examination of 2,684 B1D-only cases and 14,018 controls did not reveal an enrichment of rare P-LP variants in the BD GWAS (OR = 0.89 , one-sided $p=0.74$ ), BD MAGMA ( $\mathrm{OR}=0.98$, onesided $p=0.54$ ), or BD MAGMA-GWAS (OR $=0.85$, onesided $p=0.65$ ) gene sets (Table S7). Thus, the initial observed enrichment of rare functional protein-altering variants within BD GWAS genes was not replicated. 

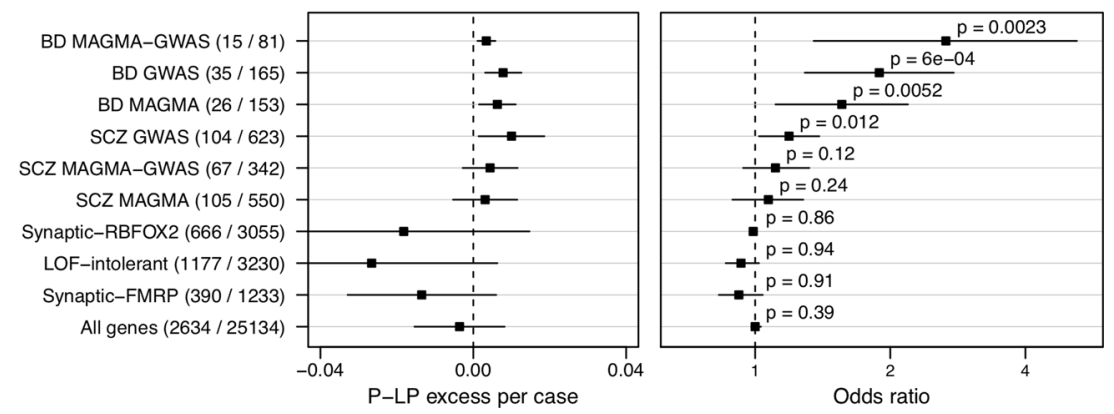

Fig. 2 P-LP variant burden in candidate BD- and SCZ-related gene sets. Meta-analysis of Firth logistic regression of P-LP variants shows that BD cases in BSC cohorts appear to carry a higher burden of P-LP alleles in three BD GWAS-derived gene sets. Horizontal bars represent $95 \%$ confidence intervals. No enrichment of P-LP variants was observed in three schizophrenia GWAS-derived gene sets, in two neuron synaptic-related genesets (RBFOX2- and FMRP-related

Given the shared clinical features and reports of shared common genetic susceptibility between BD and SCZ, we also examined the burden of rare P-LP variants for three schizophrenia-related gene sets. Meta-analysis across cohorts showed no significant enrichment of rare P-LP variants in the $623 \mathrm{SCZ}$ GWAS genes $(\mathrm{OR}=1.19,95 \% \mathrm{CI}$ $=1.02-1.39$, one-sided $p=0.01$, adjusted one-sided $p=$ $0.08)$, in $550 \mathrm{SCZ}$ MAGMA genes $(\mathrm{OR}=1.07,95 \% \mathrm{CI}=$ $0.89-1.28$, one-sided $p=0.24$ ), and in 342 SCZ MAGMAGWAS genes $(\mathrm{OR}=1.11,95 \% \mathrm{CI}=0.94-1.32$, onesided $p=0.12$ ) (Fig. 2, Tables 2, S4 and S5). CMH analysis again showed results similar to meta-analysis with Firth logistic regression (Tables 2 and S4). This suggests that despite the shared common genetic susceptibility between $\mathrm{BD}$ and $\mathrm{SCZ}$, we found no evidence for BD risk being associated with rare functional protein-altering variation in schizophrenia GWAS genes.

We examined three gene sets previously reported to harbor rare variants that influence neuropsychiatric traits: 3,055 genes whose mRNAs are bound by the synaptic protein RBFOX2, 1,233 FMRP-related genes that colocalize with neuronal synapses, and 3,230 genes reported to be intolerant to loss-of-function mutations. Each of these gene sets has been implicated in rare variant studies of schizophrenia [26, 32, 34, 50, 52]. We observed no significant enrichment of rare P-LP variants in the RBFOX2related genes (meta-analysis $\mathrm{OR}=0.99,95 \% \mathrm{CI}=$ $0.98-1.01$, one-sided $p=0.86$ ), FMRP-related genes (OR $=0.93,95 \% \mathrm{CI}=0.82-1.04$, one-sided $p=0.91$ ), or LOFintolerant genes $(\mathrm{OR}=0.94,95 \% \mathrm{CI}=0.86-1.02$, onesided $p=0.94)$ in $\mathrm{BD}$ cases versus controls across studies (Fig. 3). CMH analysis confirmed a lack of rare variant enrichment within these gene sets (Table 2), thus we had no evidence of $\mathrm{BD}$ risk from rare functional protein-altering variants in a broad set of genes encoding proteins that genes), in genes classified as LOF-intolerant, or in all genes across the exome. $P$ values are one-sided for enrichment of P-PL variants in $\mathrm{BD}$ cases and derived from the meta-analysis Z-score. Numbers in parentheses represent the number of genes with P-LP variants and total number of genes within each gene set, respectively. BD bipolar disorder, OR odds ratio, P-LP pathogenic or likely pathogenic, GWAS genome-wide association study, LOF loss-of-function.

localize to neuronal synapses, that were previously associated with schizophrenia.

Lastly, we used published PGC2 case-control GWAS meta-analysis results for $\mathrm{BD}$ and schizophrenia to test the common polygenic burden on risk of BD in our BSC subjects. Consistent with previous publications [60], common variant PRS for both $\mathrm{BD}$ and schizophrenia were highly significant in the combined meta-analysis $\left(\mathrm{p}_{\mathrm{BD}}=\right.$ $1.52 \times 10^{-41}$ and $\mathrm{p}_{\mathrm{SZ}}=1.51 \times 10^{-32}$, Table $\mathrm{S} 8$ ), but explained only a small proportions of variance in disease risk $(\sim 1-4 \%)$ in individual BSC studies, again demonstrating the shared genetic etiology of these psychiatric conditions, at least from common variants and in contrast to rare variant analyses.

\section{Discussion}

To our knowledge, this primary analysis of 3,987 BD cases and 5,322 controls, along with independent replication/second stage samples of 9,929 cases and 14,018 controls represents the largest study of coding sequence variation in BD. We examined the exomes of these individuals, and identified rare coding variants classified as P-LP according to ACMG criteria [42, 44]. We did not observe a significant enrichment of rare P-LP variants in BSC BD cases versus controls overall or for any individual gene. We also did not observe an increased burden of rare P-LP variants in genes implicated by BD GWAS regions in the combined BSC and BipEx cases. This observation diverges from studies in some other complex disorders that found an enrichment of rare coding variation in genes associated with GWAS loci identified from common variants and vice versa [19-21, 61].

We did not observe a difference in rare P-LP variant burden within gene sets that influence synaptic function 


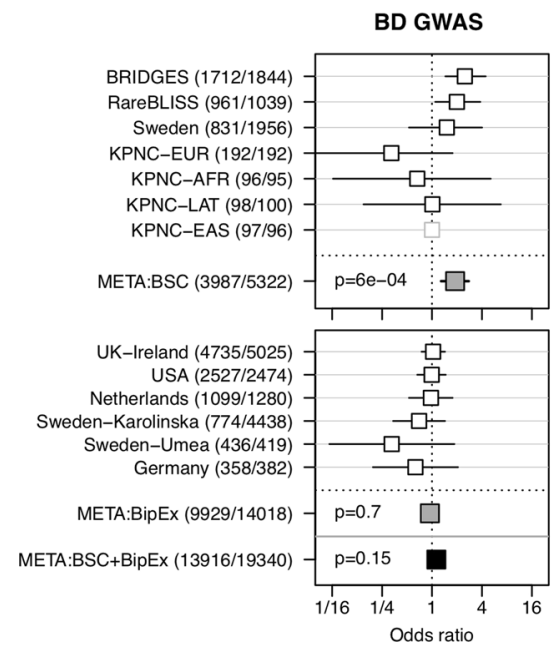

Fig. 3 P-LP variant burden in BD GWAS gene sets within BSC discovery and BipEx replication cohorts. Forest plots of log odds ratios (Firth logistic regressions) for association between P-LP variant burden and BD across seven cohorts/ethnicities in the BSC study (top) and across six strata in the BipEx replication cohort (bottom). $p$ values are one-sided for enrichment of P-PL variants in BD cases and derived from the meta-analysis Z-score. Meta-analysis shows that individuals with BD do not carry a replicable enrichment of P-LP variants in 165 BD GWAS-derived genes, in $153 \mathrm{BD}$ genes identified using MAGMA, or in 81 genes that overlap between the BD GWAS and BD

(RBFOX2 and FMRP), genes that are intolerant to loss-offunction mutations, or all genes across the exome. This diverges from prior studies of exonic variation in familial $\mathrm{BD}$ cases that suggest a role for synaptic-related genes [25], ion channels [23], and modulation of neurotransmitter activity [24]. However, those studies were small (far smaller than ours) and their findings unreplicated, highlighting the need for large studies with replication cohorts. While this study was not powered to identify novel single gene associations, it provides important insight into the genetic architecture of rare coding variants in $\mathrm{BD}$, specifically the absence of replicable enrichment of such variants in several biologically plausible gene sets.

A shared genetic susceptibility to BD and SCZ is suggested through investigations of genome-wide common variation and polygenic risk scores (PRS) [12, 27-29]. Our results confirmed the correlation of common $\mathrm{SCZ}$ variants with BD risk. Moreover, examination of sub-phenotypes suggests that the SCZ PRS is associated with psychosis in $\mathrm{BD}$, and that the BD PRS is associated with mania in SCZ $[23,24]$. However, examination of rare coding variation does not support a clear role for BD-related genes in SCZ risk [32]. Similarly, we observe no significant enrichment of rare P-LP variants within SCZ GWAS genes in individuals with $\mathrm{BD}$, or in other gene sets (RBFOX2, FMRP, LOFintolerant, exome-wide) that have been reported to harbor rare variants in SCZ $[32,35]$. While larger studies might reveal a smaller effect within these broader gene sets, this
BD MAGMA

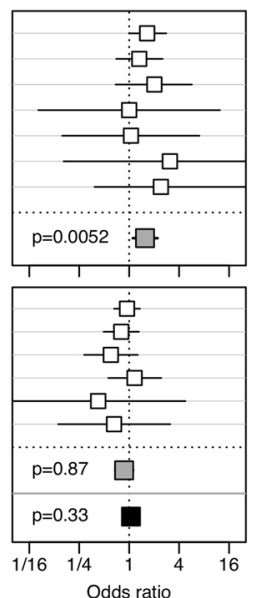

BD MAGMA-GWAS

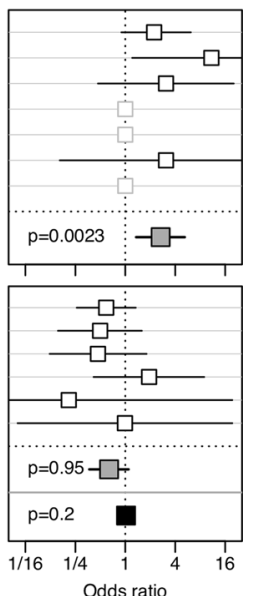

MAGMA gene sets. Horizontal black lines indicate 95\% confidence intervals around the effect size. Unshaded boxes indicate absence of $\mathrm{P}$ LP variants within a specific cohort. Gray boxes indicate meta-analysis within the BSC (discovery) and BipEx (replication) cohorts, respectively, and black box indicates meta-analysis across all BSC and BipEx cohorts. Numbers in parentheses indicate the number of BD cases and controls. BSC Bipolar Sequencing Consortium, BipEx Bipolar Exomes collection, REP replication cohorts, META metaanalysis.

study does not show a clear role for rare variants within SCZ-related genes in BD.

The absence of deleterious rare variant enrichment in $\mathrm{BD}$ cases contrasts to studies of SCZ of similar size (4-5 K cases, 7-9 K controls) which show that individuals with SCZ carry an increased burden of rare protein-truncating variants across the exome, particularly in genes intolerant to loss-of-function mutations [32, 35]. This burden was particularly elevated in SCZ cases with intellectual disability [35]. Another study showed that rare CNVs are enriched in individuals with schizoaffective disorder bipolar type (SAB), but not in all individuals with BD [62]. These observations suggest that, while there exists a shared susceptibility for BD and SCZ through common genetic variation, rare coding variation in gene sets implicated in SCZ might preferentially confer a risk for SCZ-specific features independent of the affective symptoms that are definitional of BD. Thus, individuals with rare variants in genes implicated in SCZ might present with psychotic or cognitive predominant symptoms, rather than manic or hypomanic episodes, which are required for a diagnosis of BD.

In this study, variants classified as pathogenic are loss-offunction (LOF) mutations (splice-site and stop-gain) in a gene where LOF is a known mechanism of disease, while those classified as likely pathogenic are primarily missense variants in a gene with a low rate of benign missense variation. Both types of variants are absent or at very low frequency in large control cohorts (gnomAD), and have 
Table 2 Rare variant burden in candidate gene sets.

\begin{tabular}{|c|c|c|c|c|c|c|c|}
\hline \multicolumn{5}{|l|}{ Gene sets examined } & \multicolumn{3}{|c|}{ Meta-analysis of Firth logistic regressions } \\
\hline \multirow[t]{2}{*}{ Gene set } & \multicolumn{2}{|l|}{ Genes } & \multicolumn{2}{|l|}{ P-LP count } & \multirow[t]{2}{*}{ OR $(95 \% \mathrm{CI})$} & \multirow[t]{2}{*}{ One-sided $p$ value } & \multirow[t]{2}{*}{ Adjusted $p$ value } \\
\hline & All & Carrying P-LP variants & 3987 cases & 5322 controls & & & \\
\hline BD GWAS & 165 & 35 & 78 & 51 & $1.89(1.29-2.77)$ & 0.0006 & 0.006 \\
\hline BD MAGMA & 153 & 29 & 72 & 53 & $1.56(1.11-2.19)$ & 0.0052 & 0.042 \\
\hline BD MAGMA-GWAS & 81 & 15 & 21 & 8 & $2.66(1.35-5.21)$ & 0.0023 & 0.021 \\
\hline SCZ GWAS & 623 & 111 & 288 & 299 & $1.19(1.02-1.39)$ & 0.011 & 0.077 \\
\hline SCZ MAGMA & 550 & 113 & 282 & 331 & $1.07(0.89-1.28)$ & 0.24 & 1.00 \\
\hline SCZ MAGMA-GWAS & 342 & 72 & 198 & 219 & $1.11(0.94-1.32)$ & 0.12 & 0.72 \\
\hline Synaptic-RBFOX2 & 3,055 & 698 & 1,219 & 1,555 & $0.99(0.98-1.01)$ & 0.86 & 1.00 \\
\hline Synaptic-FMRP & 1,233 & 413 & 756 & 976 & $0.92(0.83-1.04)$ & 0.91 & 1.00 \\
\hline LOF-intolerant & 3,230 & 1,246 & 1628 & 2,059 & $0.93(0.86-1.02)$ & 0.94 & 1.00 \\
\hline All genes & 25,134 & 2,634 & 7,847 & 9,787 & $1.00(0.97-1.03)$ & 0.39 & 1.00 \\
\hline
\end{tabular}

For each of 10 candidate gene sets, we performed a meta-analysis of the effect of P-LP variant burden on BD within each study (using adjusted Firth logistic regression), and calculated a one-sided $p$ value examining enrichment of P-LP variants in BD cases. Adjusted $p$ values represent correction for multiple comparisons using the Holm method.

$B D$ bipolar disorder, SCZ schizophrenia, GWAS genome-wide association study, LOF loss-of-function, $O R$ odds ratio.

multiple lines of evidence for a deleterious effect. While there are likely a number of P-LP variants not included as PLP in our study (e.g., frameshift LOF variants), their noninclusion would only have limited the power of our analysis, and not created a bias. Nonetheless, examination of the P-LP variants we included did enable systematic interrogation of candidate gene sets that might be biologically relevant for a complex phenotype such as BD.

Although we found three significantly associated genesets in our BSC study, these genesets did not replicate in the BipEx study and they were not significant in a metaanalysis of the BSC and BipEx studies. There are differences in the case composition of the BSC and BipEx samples. More BD cases were classified as B1D, a form of $\mathrm{BD}$ with more extreme episodes of mood elevation [63], in the BSC discovery cohort (91\% of cases) than in the BipEx replication cohort (74\% of cases with known subtypes), However, when restricting to the smaller set of BipEx B1D cases we still did not detect association in the genesets identified in the BSC studies. It may be that the BSC geneset-based results are false positives or that larger sample sizes with deleterious variants defined by other metrics may be necessary to consistently detect a signal.

We acknowledge a number of limitations. First, there are a number of inter-study differences in methods used for case-control selection, DNA sample acquisition, exome or genome sequencing, and data quality control. While these differences might contribute to biases in variant detection in cases and controls, we showed that our approach produced no exome-wide deviation of rare synonymous variants in cases versus controls. Second, our analysis was limited to coding single nucleotide variants, which have the highest quality annotation in large public databases (gnomAD) and annotation tools (InterVar). Future WGS studies that include analysis of copy number, insertion-deletion, and regulatory variants may shed further light on the role of rare variants in BD. Third, while genome-wide common variant studies implicate loci that influence susceptibility for BD, many genes in these regions that were included in our analysis are likely unrelated to $\mathrm{BD}$, highlighting the need for fine-mapping and functional studies. Lastly, our ability to further investigate the observed differences between the discovery and replication cohorts was limited due to the lack of deep sub-phenotype data (such as severity of mood episodes, psychosis, hospitalizations) in the majority of BD cases.

Despite the limitations, this study provides important biologic insight into disease pathogenesis in BD. While common and rare variant associations implicate several genes critical for neuronal development and synaptic function [15-18, 22-26], this study suggests that BD risk may not be broadly influenced by rare coding variation in genes within these categories. Moreover, while common genetic association studies suggest a role for shared genetic susceptibility between BD and SCZ, there is limited evidence for a shared susceptibility from rare genetic variation for these related mental disorders. Additional studies are needed to dissect the relationship between rare coding variants and subtypes of $\mathrm{BD}$, including SAB. Neuroimaging and neurophysiological biomarkers $[64,65]$ may be used to identify genetic factors that influence endophenotypes within these complex 
disorders. Lastly, loci identified through BD common variant studies likely require fine-mapping through functional studies, and not only through scalable genomics methods, for translational impact.

Acknowledgements Funding for this study is from: International Bipolar Sequencing Consortium (NIMH R01 MH 110437) (P.Z. M.B., J.P., N.F., P.S.); Whole Genome Sequencing for Schizophrenia and Biopolar Disorder in the GPS (NIMH UO1 MH105653) (M.B, R.M. M.); Whole Genome and Exome Sequencing for Bipolar Disorder (NIMH R01 MH 094145) (M.B.); Multi-ethnic GWAS of Bipolar I Disorder (NIH R01 MH 085543) (C.S.); Genetic Epidemiology Research in Adult Health and Aging (GERA RC2 AG036607) (C.S., N.R.) from the Kaiser Permanente Research Program on Genes, Environment, and Health; Rare Bipolar Loci Identification Through Synaptome Sequencing (NIMH R01 MH 087979 and MH 087992) (J.P., W.R.M); 2/2 Large Scale Genetic Studies of Schizophrenia in Sweden (R01MH095034) (E.A.S.); 1/3 Genetic Analysis of the International Cohort Collection for Bipolar Disorder (R01MH106531) (E.A.S.); 1/2 Large Scale Genetic Studies of Schizophrenia in Sweden (R01MH077139) (P.F.S.); 2/3 Genetic Analysis of the International Cohort Collection for Bipolar Disorder (R01MH106527) (S.A.M.); The Dalio Foundation (B.N.).

\section{Compliance with ethical standards}

Conflict of interest $\mathrm{XJ}$ had no conflicts during the time he contributed to this study, and declares he is now an employee at Genentech. AEL and EAS had no conflicts during the time they contributed to this study, and declare they are now employees at Regeneron. ML declares that, over the past 36 months, he has received lecture honoraria from Lundbeck pharmaceutical. CL is a member of the Myriad Neuroscience R\&D SAB. BN is a Deep Genomics-Member, Scientific Advisory Board; Camp4 Therapeutics Corporation-Consultant, Scientific Advisory Board; Takeda Pharmaceutical-Consultant; Biogen-Consultant, Genomics Analytics Advisory Panel. MO has a research grant from Takeda Pharmaceuticals. JS is an unpaid member of the Bipolar/ Depression Research Community Advisory Panel of 23andMe.

Publisher's note Springer Nature remains neutral with regard to jurisdictional claims in published maps and institutional affiliations.

Open Access This article is licensed under a Creative Commons Attribution 4.0 International License, which permits use, sharing, adaptation, distribution and reproduction in any medium or format, as long as you give appropriate credit to the original author(s) and the source, provide a link to the Creative Commons licence, and indicate if changes were made. The images or other third party material in this article are included in the article's Creative Commons licence, unless indicated otherwise in a credit line to the material. If material is not included in the article's Creative Commons licence and your intended use is not permitted by statutory regulation or exceeds the permitted use, you will need to obtain permission directly from the copyright holder. To view a copy of this licence, visit http://creativecommons. org/licenses/by/4.0/.

\section{References}

1. Ferrari AJ, Stockings E, Khoo JP, Erskine HE, Degenhardt L, Vos $\mathrm{T}$, et al. The prevalence and burden of bipolar disorder: findings from the Global Burden of Disease Study 2013. Bipolar Disord. 2016;18:440-50.
2. Merikangas KR, Jin R, He J-P, Kessler RC, Lee S, Sampson NA, et al. Prevalence and correlates of bipolar spectrum disorder in the world mental health survey initiative. Arch Gen Psychiatry. 2011;68:241-51.

3. Grande I, Berk M, Birmaher B, Vieta E. Bipolar disorder. Lancet. 2016;387:1561-72.

4. Beasley CL, Honer WG, von Bergmann K, Falkai P, Lütjohann D, Bayer TA. Reductions in cholesterol and synaptic markers in association cortex in mood disorders. Bipolar Disord. 2005;7:449-55.

5. Martinowich K, Schloesser RJ, Manji HK. Bipolar disorder: from genes to behavior pathways. J Clin Investig. 2009;119:726-36.

6. Vawter MP, Thatcher L, Usen N, Hyde TM, Kleinman JE, Freed WJ. Reduction of synapsin in the hippocampus of patients with bipolar disorder and schizophrenia. Mol Psychiatry. 2002;7:571-78.

7. Konopaske GT, Lange N, Coyle JT, Benes FM. Prefrontal cortical dendritic spine pathology in schizophrenia and bipolar disorder. JAMA Psychiatry. 2014;71:1323-31.

8. Berk M, Kapczinski F, Andreazza AC, Dean OM, Giorlando F, Maes M, et al. Pathways underlying neuroprogression in bipolar disorder: focus on inflammation, oxidative stress and neurotrophic factors. Neurosci Biobehav Rev. 2011;35:804-17.

9. Rakofsky JJ, Ressler KJ, Dunlop BW. BDNF function as a potential mediator of bipolar disorder and post-traumatic stress disorder comorbidity. Mol Psychiatry. 2012;17:22-35.

10. Craddock N, Sklar P. Bipolar disorder 1 - genetics of bipolar disorder. Lancet. 2013;381:1654-62.

11. Kieseppä T, Partonen T, Haukka J, Kaprio J, Lönnqvist J. High concordance of bipolar I disorder in a nationwide sample of twins. Am J Psychiatry. 2004;161:1814-21.

12. Lichtenstein P, Yip BH, Bjork C, Pawitan Y, Cannon TD, Sullivan $\mathrm{PF}$, et al. Common genetic determinants of schizophrenia and bipolar disorder in Swedish families: a population-based study. Lancet. 2009;373:234-39.

13. McGuffin P, Rijsdijk F, Andrew M, Sham P, Katz R, Cardno A. The heritability of bipolar affective disorder and the genetic relationship to unipolar depression. Arch Gen Psychiatry. 2003;60:497-502.

14. Smoller JW, Finn CT. Family, twin, and adoption studies of bipolar disorder. Am J Med Genet Part C. 2003;123c:48-58.

15. Chen DT, Jiang X, Akula N, Shugart YY, Wendland JR, Steele $\mathrm{CJ}$, et al. Genome-wide association study meta-analysis of European and Asian-ancestry samples identifies three novel loci associated with bipolar disorder. Mol Psychiatry. 2013;18:195205.

16. Group PGCBDW. Large-scale genome-wide association analysis of bipolar disorder identifies a new susceptibility locus near ODZ4. Nat Genet. 2011;43:977-83.

17. Muhleisen TW, Leber M, Schulze TG, Strohmaier J, Degenhardt F, Treutlein J, et al. Genome-wide association study reveals two new risk loci for bipolar disorder. Nat Commun. 2014;5:3339.

18. Stahl EA, Breen G, Forstner AJ, McQuillin A, Ripke S, Trubetskoy V, et al. Genome-wide association study identifies 30 loci associated with bipolar disorder. Nat Genet. 2019;51:793-803.

19. Fuchsberger C, Flannick J, Teslovich TM, Mahajan A, Agarwala $\mathrm{V}$, Gaulton KJ, et al. The genetic architecture of type 2 diabetes. Nature. 2016;536:41-47.

20. Consortium IMS. Low-frequency and rare-coding variation contributes to multiple sclerosis risk. Cell. 2018;175:1679-87.e1677.

21. Visscher PM, Wray NR, Zhang Q, Sklar P, McCarthy MI, Brown MA, et al. 10 Years of GWAS discovery: biology, function, and translation. Am J Hum Genet. 2017;101:5-22.

22. Kataoka M, Matoba N, Sawada T, Kazuno AA, Ishiwata M, Fujii $\mathrm{K}$, et al. Exome sequencing for bipolar disorder points to roles of 
de novo loss-of-function and protein-altering mutations. Mol Psychiatry. 2016;21:885-93.

23. Ament SA, Szelinger S, Glusman G, Ashworth J, Hou L, Akula $\mathrm{N}$, et al. Rare variants in neuronal excitability genes influence risk for bipolar disorder. Proc Natl Acad Sci USA. 2015;112:3576.

24. Lescai F, Als TD, Li Q, Nyegaard M, Andorsdottir G, Biskopst $\varnothing$ $\mathrm{M}$, et al. Whole-exome sequencing of individuals from an isolated population implicates rare risk variants in bipolar disorder. Transl Psychiatry. 2017;7:e1034.

25. Toma C, Shaw AD, Allcock RJN, Heath A, Pierce KD, Mitchell $\mathrm{PB}$, et al. An examination of multiple classes of rare variants in extended families with bipolar disorder. Transl Psychiatry. 2018;8:65.

26. Goes FS, Pirooznia M, Parla JS, Kramer M, Ghiban E, Mavruk S, et al. Exome sequencing of familial bipolar disorder. JAMA Psychiatry. 2016;39:139-55.

27. Mortensen PB, Pedersen CB, Melbye M, Mors O, Ewald H. Individual and familial risk factors for bipolar affective disorders in Denmark. Arch Gen Psychiatry. 2003;60:1209-15.

28. Ruderfer DM, Ripke S, McQuillin A, Boocock J, Stahl EA, Pavlides JMW, et al. Genomic dissection of bipolar disorder and schizophrenia, including 28 subphenotypes. Cell. 2018;173:170515.e16.

29. Van Snellenberg JX, De Candia T. Meta-analytic evidence for familial coaggregation of schizophrenia and bipolar disorder. Arch Gen Psychiatry. 2009;66:748-55.

30. Cross-Disorder Group of the Psychiatric Genomics C, Lee SH, Ripke S, Neale BM, Faraone SV, Purcell SM, et al. Genetic relationship between five psychiatric disorders estimated from genome-wide SNPs. Nat Genet. 2013;45:984-94.

31. Ruderfer DM, Fanous AH, Ripke S, McQuillin A, Amdur RL, Gejman PV, et al. Polygenic dissection of diagnosis and clinical dimensions of bipolar disorder and schizophrenia. Mol Psychiatry. 2014;19:1017-24.

32. Genovese G, Fromer M, Stahl EA, Ruderfer DM, Chambert K, Landén $\mathrm{M}$, et al. Increased burden of ultra-rare protein-altering variants among 4,877 individuals with schizophrenia. Nat Neurosci. 2016;19:1433-41.

33. Kenny EM, Cormican P, Furlong S, Heron E, Kenny G, Fahey C, et al. Excess of rare novel loss-of-function variants in synaptic genes in schizophrenia and autism spectrum disorders. Mol Psychiatry. 2014;19:872-9.

34. Purcell SM, Moran JL, Fromer M, Ruderfer D, Solovieff N, Roussos $\mathrm{P}$, et al. A polygenic burden of rare disruptive mutations in schizophrenia. Nature. 2014;506:185-90.

35. Singh T, Walters JTR, Johnstone M, Curtis D, Suvisaari J, Torniainen $\mathrm{M}$, et al. The contribution of rare variants to risk of schizophrenia in individuals with and without intellectual disability. Nat Genet. 2017;49:1167.

36. Ganna A, Satterstrom FK, Zekavat SM, Das I, Kurki MI, Churchhouse C, et al. Quantifying the impact of rare and ultra-rare coding variation across the phenotypic spectrum. Am J Hum Genet. 2018;102:1204-11.

37. Carlson J, Locke AE, Flickinger M, Zawistowski M, Levy S, Myers RM, et al. Extremely rare variants reveal patterns of germline mutation rate heterogeneity in humans. Nat Commun. 2018;9:3753.

38. Banda Y, Kvale MN, Hoffmann TJ, Hesselson SE, Ranatunga D, Tang $\mathrm{H}$, et al. Characterizing race/ethnicity and genetic ancestry for 100,000 subjects in the genetic epidemiology research on adult health and aging (GERA) cohort. Genetics. 2015;200:1285-95.

39. Li H, Durbin R. Fast and accurate short read alignment with Burrows-Wheeler transform. Bioinforma. 2009;25:1754-60.

40. Jun G, Wing MK, Abecasis GR, Kang HM. An efficient and scalable analysis framework for variant extraction and refinement from population-scale DNA sequence data. Genome Res. 2015;25:918-25.

41. McKenna A, Hanna M, Banks E, Sivachenko A, Cibulskis K, Kernytsky A, et al. The genome analysis toolkit: a MapReduce framework for analyzing next-generation DNA sequencing data. Genome Res. 2010;20:1297-303.

42. Li Q, Wang K. InterVar: clinical interpretation of genetic variants by the 2015 ACMG-AMP guidelines. Am J Hum Genet. 2017;100:267-80.

43. Yang H, Wang K. Genomic variant annotation and prioritization with ANNOVAR and wANNOVAR. Nat Protoc. 2015;10:155666.

44. Richards S, Aziz N, Bale S, Bick D, Das S, Gastier-Foster J, et al. Standards and guidelines for the interpretation of sequence variants: a joint consensus recommendation of the American College of Medical Genetics and Genomics and the Association for Molecular Pathology. Genet Med. 2015;17:405-24.

45. Lek M, Karczewski KJ, Minikel EV, Samocha KE, Banks E, Fennell T, et al. Analysis of protein-coding genetic variation in 60,706 humans. Nature. 2016;536:285-91.

46. Karczewski KJ, Francioli LC, Tiao G, Cummings BB, Alföldi J, Wang $\mathrm{Q}$, et al. The mutational constraint spectrum quantified from variation in 141,456 humans. Nature. 2020;581:434-43.

47. de Leeuw CA, Mooij JM, Heskes T, Posthuma D. MAGMA: generalized gene-set analysis of GWAS data. PLoS Comput Biol. 2015;11:e1004219.

48. Pardiñas AF, Holmans P, Pocklington AJ, Escott-Price V, Ripke $\mathrm{S}$, Carrera $\mathrm{N}$, et al. Common schizophrenia alleles are enriched in mutation-intolerant genes and in regions under strong background selection. Nat Genet. 2018;50:381-9.

49. Satterstrom FK, Walters RK, Singh T, Wigdor EM, Lescai F, Demontis D, et al. ASD and ADHD have a similar burden of rare protein-truncating variants. Nat Neurosci. 2019;22:1961-5.

50. Fromer M, Pocklington AJ, Kavanagh DH, Williams HJ, Dwyer $\mathrm{S}$, Gormley $\mathrm{P}$, et al. De novo mutations in schizophrenia implicate synaptic networks. Nature. 2014;506:179-84.

51. Weyn-Vanhentenryck SM, Mele A, Yan Q, Sun S, Farny N, Zhang Z, et al. HITS-CLIP and integrative modeling define the rbfox splicing-regulatory network linked to brain development and autism. Cell Reports. 2014;6:1139-52.

52. Darnell JC, Van Driesche SJ, Zhang C, Hung KYS, Mele A, Fraser CE, et al. FMRP stalls ribosomal translocation on mRNAs linked to synaptic function and autism. Cell. 2011;146:247-61.

53. R Development Core Team. R: A language and environment for statistical computing. R Foundation for Statistical Computing: Vienna, Australia, 2010.

54. Hoffmann TJ, Kvale MN, Hesselson SE, Zhan Y, Aquino C, Cao $\mathrm{Y}$, et al. Next generation genome-wide association tool: design and coverage of a high-throughput European-optimized SNP array. Genomics. 2011;98:79-89.

55. Smith EN, Bloss CS, Badner JA, Barrett T, Belmonte PL, Berrettini $\mathrm{W}$, et al. Genome-wide association study of bipolar disorder in European American and African American individuals. Mol Psychiatry. 2009;14:755-63.

56. Smith EN, Koller DL, Panganiban C, Szelinger S, Zhang P, Badner JA, et al. Genome-wide association of bipolar disorder suggests an enrichment of replicable associations in regions near genes. PLoS Genet. 2011;7:e1002134.

57. Hoffmann TJ, Zhan Y, Kvale MN, Hesselson SE, Gollub J, Iribarren $\mathrm{C}$, et al. Design and coverage of high throughput genotyping arrays optimized for individuals of East Asian, African American, and Latino race/ethnicity using imputation and a novel hybrid SNP selection algorithm. Genomics. 2011;98:422-30.

58. Kvale MN, Hesselson S, Hoffmann TJ, Cao Y, Chan D, Connell S, et al. Genotyping Informatics and Quality Control for 100,000 
Subjects in the Genetic Epidemiology Research on Adult Health and Aging (GERA) Cohort. Genetics. 2015;200:1051-60.

59. Schizophrenia Working Group of the Psychiatric Genomics C. Biological insights from 108 schizophrenia-associated genetic loci. Nature. 2014;511:421-7.

60. Psychiatric GCBDWG. Large-scale genome-wide association analysis of bipolar disorder identifies a new susceptibility locus near ODZ4. Nat Genet. 2011;43:977-83.

61. Hoffmann TJ, Theusch E, Haldar T, Ranatunga DK, Jorgenson E, Medina MW, et al. A large electronic-health-record-based genome-wide study of serum lipids. Nat Genet. 2018;50:401-13.

62. Charney AW, Stahl EA, Green EK, Chen C-Y, Moran JL, Chambert K, et al. Contribution of rare copy number variants to bipolar disorder risk is limited to schizoaffective cases. Biological Psychiatry. 2018;86:110-9.

63. Baldessarini RJ, Undurraga J, Vázquez GH, Tondo L, Salvatore $\mathrm{P}$, $\mathrm{Ha} \mathrm{K}$, et al. Predominant recurrence polarity among 928 adult international bipolar I disorder patients. Acta Psychiatrica Scandinavica. 2012;148:129-35.

64. Koutsouleris N, Meisenzahl EM, Borgwardt S, Riecher-Rössler A, Frodl T, Kambeitz J, et al. Individualized differential diagnosis of schizophrenia and mood disorders using neuroanatomical biomarkers. Brain: a J Neurol. 2015;138:2059-73.

65. Bellivier F, Geoffroy PA, Scott J, Schurhoff F, Leboyer M, Etain B. Biomarkers of bipolar disorder: specific or shared with schizophrenia? Front Biosci. 2013;5:845-63.

\section{Affiliations}

Xiaoming Jia ${ }^{1} \cdot$ Fernando S. Goes $\mathbb{1}^{2} \cdot$ Adam E. Locke $\mathbb{1}^{3} \cdot$ Duncan Palmer $\mathbb{D}^{4} \cdot$ Weiqing Wang $\mathbb{( 1 )}^{5}$. Sarah Cohen-Woods $s^{6,7} \cdot$ Giulio Genovese $\mathbb{1}^{4} \cdot$ Anne U. Jackson $\mathbb{1}^{8} \cdot$ Chen Jiang $^{9} \cdot$ Mark Kvale $^{10} \cdot$ Niamh Mullins $^{11}$. Hoang Nguyen $\mathbb{1}^{5} \cdot$ Mehdi Pirooznia $\mathbb{1}^{12} \cdot$ Margarita Rivera ${ }^{7,13} \cdot$ Douglas M. Ruderfer ${ }^{14} \cdot$ Ling Shen $^{9} \cdot$ Khanh Thai $^{9}$. Matthew Zawistowski $\mathbb{\Phi}^{8}$. Yongwen Zhuang ${ }^{8} \cdot$ Gonçalo Abecasis $\mathbb{1}^{8} \cdot$ Huda Akil ${ }^{15}$. Sarah Bergen $\mathbb{1}^{16}$. Margit Burmeister $\mathbb{1}^{15,17,18,19}$. Sinéad Chapman ${ }^{4} \cdot$ Melissa DelaBastide $^{20} \cdot$ Anders Juréus $^{16} \cdot$ Hyun Min Kang $^{8}$. Pui-Yan Kwok $\mathbb{D}^{10} \cdot$ Jun Z. Li $\mathbb{1}^{17,18} \cdot$ Shawn E. Levy $\mathbb{1}^{21} \cdot$ Eric T. Monson ${ }^{22} \cdot$ Jennifer Moran ${ }^{23} \cdot$ Janet Sobell $^{24}$. Stanley Watson $\mathbb{1}^{15} \cdot$ Virginia Willour ${ }^{22}$. Sebastian Zöllner ${ }^{8,19} \cdot$ Rolf Adolfsson ${ }^{25} \cdot$ Douglas Blackwood $\mathbb{C}^{26}$.

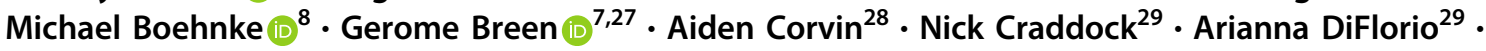
Christina M. Hultman ${ }^{16} \cdot$ Mikael Landen $\mathbb{1 0}^{16,30}$ - Cathryn Lewis $\mathbb{1}^{7,31} \cdot$ Steven A. McCarroll $\mathbb{1}^{32}$ • W. Richard McCombie ${ }^{20} \cdot$ Peter McGuffin ${ }^{7}$. Andrew Mclntosh $\mathbb{1}^{26,33} \cdot$ Andrew McQuillin $\mathbb{1}^{34} \cdot$ Derek Morris $\mathbb{1}^{28,35}$. Richard M. Myers ${ }^{21} \cdot$ Michael O'Donovan $\mathbb{1}^{29} \cdot$ Roel Ophoff ${ }^{36,37} \cdot$ Marco Boks $\mathbb{1}^{37} \cdot$ Rene Kahn $\mathbb{1}^{38}$. Willem Ouwehand $\mathbb{1 0}^{39} \cdot$ Michael Owen $\mathbb{1}^{29} \cdot$ Carlos Pato ${ }^{24,40} \cdot$ Michele Pato ${ }^{24,41}$. Danielle Posthuma ${ }^{42,43}$. James B. Potash $\mathbb{1}^{2}$. Andreas Reif $\mathbb{1}^{44} \cdot$ Pamela Sklar ${ }^{5}$. Jordan Smoller ${ }^{4,45} \cdot$ Patrick F. Sullivan $^{46}$. John Vincent $\mathbb{C}^{47,48} \cdot$ James Walters ${ }^{29} \cdot$ Benjamin Neale $\mathbb{C}^{4,49}$. Shaun Purcell $\mathbb{C}^{50,51} \cdot$ Neil Risch $^{10}$. Catherine Schaefer $\mathbb{C}^{9} \cdot$ Eli A. Stahl $\mathbb{D}^{5} \cdot$ Peter P. Zandi $\mathbb{1}^{2} \cdot$ Laura J. Scott $\mathbb{C}^{8}$

1 Weill Institute for Neurosciences, University of California San Francisco, San Francisco, CA 94158, USA

2 Department of Psychiatry and Behavioral Sciences, Johns Hopkins School of Medicine, Baltimore, MD 21287, USA

3 Division of Genomics \& Bioinformatics, Department of Medicine and McDonnell Genome Institute, Washington University School of Medicine, St. Louis, MO 63108, USA

4 Stanley Center for Psychiatric Research, Broad Institute of MIT and Harvard, Cambridge, MA 02142, USA

5 Division of Psychiatric Genomics, Icahn School of Medicine at Mount Sinai, New York, NY 10029, USA

6 Discipline of Psychology and Flinders Centre for Innovation in Cancer, Flinders University, Adelaide, SA, Australia

7 Medical Research Council Social Genetic and Developmental Psychiatry Centre, Institute of Psychiatry, Psychology and Neuroscience, King's College London, London, UK

8 Department of Biostatistics and Center for Statistical Genetics, University of Michigan, Ann Arbor, MI 48109, USA

9 Division of Research, Kaiser Permanente Northern California, Oakland, CA 94611, USA
10 Institute for Human Genetics, University of California San Francisco, San Francisco, CA 94143, USA

11 Pamela Sklar Division of Psychiatric Genomics, Department of Genetics and Genomic Science, Icahn School of Medicine at Mount Sinai, New York, NY 10029, USA

12 Bioinformatics and Computational Core, National Heart, Lung, and Blood Institute, Bethesda, MD 20892, USA

13 Department of Biochemistry and Molecular Biology II, Institute of Neurosciences, Center for Biomedical Research, University of Granada, Granada, Spain

14 Departments of Medicine, Psychiatry, and Biomedical Informatics, Vanderbilt University Medical Center, Nashville, TN 37232 , USA

15 Molecular \& Behavioral Neuroscience Institute, University of Michigan, Ann Arbor, MI 48109, USA

16 Department of Medical Epidemiology and Biostatistics, Karolinska Institutet, Stockholm, Sweden

17 Department of Computational Medicine \& Bioinformatics, University of Michigan, Ann Arbor, MI 48109, USA

18 Department of Human Genetics, University of Michigan, Ann Arbor, MI 48109, USA 
Department of Psychiatry, University of Michigan, Ann Arbor, MI 48109, USA

20 Division of Research, Cold Spring Harbor Laboratory, Cold Spring, Harbor, NY 11797, USA

21 HudsonAlpha Institute for Biotechnology, Huntsville, AL 35806, USA

Department of Psychiatry, University of Iowa Carver College of Medicine, Iowa City, IA 52242, USA

Department of Psychiatry, Massachusetts General Hospital, Boston, MA 02114, USA

24 Department of Psychiatry and Behavioral Sciences, University of Southern California, Los Angeles, CA 90033, USA

Departments of Clinical Sciences and Psychiatry, Umea University, Umea, Sweden

26 Division of Psychiatry, University of Edinburgh, Edinburgh, UK

27 NIHR BRC for Mental Health, King's College London, London, UK

28 Department of Psychiatry and Trinity Translational Medicine Institute, Trinity College Dublin, Dublin, Ireland

29 Medical Research Council Centre for Neuropsychiatric Genetics and Genomics, Division of Psychological Medicine and Clinical Neurosciences, Cardiff University School of Medicine, Cardiff, UK

30 Institute of Neuroscience and Physiology, University of Gothenburg, Gothenburg, Sweden

31 Department of Medical \& Molecular Genetics, King's College London, London, UK

32 Department of Genetics, Harvard Medical School, Boston, MA 02115, USA

33 Centre for Cognitive Ageing and Cognitive Epidemiology, University of Edinburgh, Edinburgh, UK

34 Division of Psychiatry, University College London, London, UK

35

Discipline of Biochemistry, Neuroimaging and Cognitive Genomics (NICOG) Centre, National University of Ireland Galway, Galway, Ireland
36 Center for Neurobehavioral Genetics, University of California Los Angeles, Los Angeles, CA 90095, USA

37 Department of Psychiatry, UMC Utrecht Brain Center Rudolf Magnus, Utrecht, the Netherlands

38 Department of Psychiatry, Icahn School of Medicine at Mount Sinai, New York, NY 10029, USA

39 Department of Haematology, School of Clinical Medicine, University of Cambridge, Cambridge, UK

40 SUNY Downstate Medical Center, Brooklyn, NY 11203, USA

41 Department of Psychiatry, SUNY Downstate Medical Center, Brooklyn, NY 11203, USA

42 Department of Complex Trait Genetics, Center for Neurogenomics and Cognitive Research, Amsterdam Neuroscience, Vrije Universiteit Amsterdam, Amsterdam, the Netherlands

43 Department of Clinical Genetics, Amsterdam Neuroscience, Vrije Universiteit Medical Center, Amsterdam, the Netherlands

44 Department of Psychiatry, Psychosomatic Medicine and Psychotherapy, University Hospital Frankfurt, Frankfurt am Main, Germany

45 Department of Psychiatric and Neurodevelopmental Genetics Unit, Massachusetts General Hospital, Boston, MA 02114, USA

46 Departments of Genetics and Psychiatry, University of North Carolina, Chapel Hill, NC, USA

47 Molecular Neuropsychiatry and Development Laboratory, Campbell Family Mental Health Research Institute, Center for Addiction \& Mental Health, Toronto, ON, Canada

48 Department of Psychiatry and Institute of Medical Sciences, University of Toronto, Toronto, ON, Canada

49 Analytical and Translational Genetics Unit, Department of Medicine, Massachusetts General Hospital, Boston, MA 02114, USA

50 Division of Sleep and Circadian Disorders, Brigham and Women's Hospital and Harvard Medical School, Boston, MA 02115, USA

51 Program in Medical and Population Genetics, Broad Institute of MIT and Harvard, Cambridge, MA 02142, USA 